\title{
Lung clearance index in adult cystic fibrosis patients: the role of convection-dependent lung units
}

\author{
Sylvia Verbanck', Manuel Paiva², Erika Paeps³ ${ }^{3}$ Daniel Schuermans, \\ Anne Malfroot ${ }^{3}$, Walter Vincken ${ }^{1}$ and Eef Vanderhelst ${ }^{1}$
}

Affiliations: ${ }^{1}$ Respiratory Division, University Hospital UZ Brussel, Brussels, ${ }^{2}$ Respiratory Division, University Hospital Erasme, Université Libre de Bruxelles, Brussels, ${ }^{3}$ Cystic Fibrosis Center, University Hospital UZ Brussel, Brussels, Belgium.

Correspondence: S. Verbanck, Respiratory Division, University Hospital UZ Brussel, Laarbeeklaan 101, 1090 Brussels, Belgium. E-mail: sylvia.verbanck@uzbrussel.be

ABSTRACT We investigated the extent of convective ventilation heterogeneity contributing to the observed lung clearance index values in adult cystic fibrosis patients, as the result of two simulated scenarios, either 1) a fixed part of the lungs becoming increasingly less compliant, or 2) an increasingly greater part of the lung being less compliant.

In 25 cystic fibrosis patients and 25 matched controls, we computed the lung clearance index and also quantified curvilinearity of the washout concentration curve, where curvilinearity is equal to 0 (linear in semilog plot) reflects homogeneous ventilation and curvilinearity equal to 1 corresponds to the presence of an infinitesimally slowly emptying lung unit.

In the cystic fibrosis group (forced expiratory volume in $1 \mathrm{~s} 27-100 \%$ predicted), lung clearance index and curvilinearity average \pm SD values were $10.3 \pm 2.3$ and $0.57 \pm 0.13$, respectively, and were significantly different from control values $(6.2 \pm 0.4$ and $0.18 \pm 0.07$; both $\mathrm{p}<0.001)$; lung clearance index and curvilinearity were also correlated $(\mathrm{R}=0.67 ; \mathrm{p}<0.001)$. The average curvilinearity value in the cystic fibrosis group was found to be compatible with a cumulative volume of underventilated lung of $40-50 \%$, depending on the simulation scenario considered.

The degree of washout curvilinearity observed here indicates that a major determinant of the abnormal lung clearance index values observed in adult cystic fibrosis patients is ventilation heterogeneity generated between convection-dependent lung units, while the remainder of lung clearance index abnormality with respect to normal controls potentially represents the small airways within these lung zones.

@ERSpublications

A major determinant of the lung clearance index can be identified by considering washout curvilinearity. http://ow.ly/lAZUa

This article has supplementary material available from www.erj.ersjournals.com

Received: Aug 092012 | Accepted after revision: Oct 132012 | First published online: Oct 252012

Support statement: This study was funded by the Fund for Scientific Research, Flanders, Belgium.

Conflict of interest: None declared.

Copyright @ERS 2013 


\section{Introduction}

The lung clearance index (LCI) has been promoted for its early detection capabilities [1-3] and longitudinal studies have shown its predictive potential at preschool age [4] and progression into early adulthood [5]. Normal LCI values depend on either the washout gas (either sulfur hexafluoride or nitrogen) or concentration (mean expired or end-tidal) used for its computation, and the most commonly reported LCI based on end-tidal sulfur hexafluoride concentrations typically ranges from 5.5 to 7.5 in normal subjects and is poorly dependent on age [2]. The magnitude of LCI response to treatment intervention in cystic fibrosis (CF) patients has been slightly underwhelming. A short-acting $\beta_{2}$-agonist [6] or short-term physiotherapy [7] did not result in significant LCI change. In CF patients with an acute pulmonary exacerbation, a course of i.v. antibiotic treatment induced a mere 0.5 LCI decrease, reduced from an average baseline LCI of 10 [8]. In mild CF patients (forced expiratory volume in $1 \mathrm{~s}$ (FEV1) $>80 \%$ predicted), dornase alfa [9] and hypertonic saline [10] led to LCI decreases of 0.5-1 in patients with baseline LCI values exceeding eight. In the latter two studies, LCI decreases were not correlated to FEV1 increases, yet LCI was seen to correlate with FEV1 at baseline $[9,10]$. In advanced CF, LCI is invariably seen to be correlated with FEV1 [2, 11] and a high FEV1 is shown be a risk factor for subsequent lung function decline [12]. Taken together, these observations have regularly prompted the suggestion that LCI is not very useful in established CF lung disease and that FEV1 may in fact be the preferred outcome parameter $[1,3,10,13]$.

Besides LCI utility as compared with spirometry, its utility in relation to computed tomography imaging has also been questioned. In the absence of a gold standard for actual presence of CF lung disease, existing studies have led to paradoxical recommendations: that as long as LCI is normal there is no need for highresolution CT (HRCT) [14] or, alternatively, that HRCT should only be performed if LCI is normal [15]. The choice between these two options will depend on whether early detection or follow-up of disease is the purpose, but is also hindered by the fact that the meaning of abnormal LCI values frequently observed in CF has been elusive. Relationships between LCI and CT scores of bronchiectasis have been found [15, 16], and CT studies of disease progression have identified a marked contribution from peripheral bronchiectasis to worsening overall CT scores in children but not in adults [17]. Hence, LCI in adult CF is likely to be the composite result of potentially irreversible structural abnormalities at all length scales down to the periphery, also including peripheral structures beyond CT resolution, as inferred from tests of acinar ventilation heterogeneity in school-aged children, adolescents and adults [6, 18-20].

In principle, LCI is a combined result of simple dilution, serial dead space (including instrumental and anatomical dead space), specific ventilation differences between parallel lung units of various sizes (supplied by convective flow) and physiological dead space generated by intra-acinar ventilation heterogeneities (due to a diffusion-convection interaction) [21,22]. Estimating the contribution of the latter effect to LCI with the standard phase III slope analysis [20] is complicated in cases of severe ventilation heterogeneity. However, in such extreme cases, the washout concentration curve, and its curvilinearity in particular, which is less affected by acinar effects [22], is likely to provide a large signal that can be exploited to actually estimate the proportion of lung unit that is responsible for generating convection-dependent ventilation heterogeneities. In the present study, we have taken up this challenge and estimated by a simple calculation what amount of poorly ventilated lung would be compatible with experimental washout data. We hypothesise that the LCI values obtained in adult CF are determined in a large part by convectiondependent specific ventilation heterogeneities between units cumulatively representing a considerable portion of total lung volume.

\section{Materials and methods}

The study protocol was approved by the local research ethics committee (UZ Brussels ethics committee; Belgian unique number 143201213405). We studied 25 consecutive adult CF patients, proven by sweat chloride testing and genetic typing, in a stable clinical setting. We also recruited 25 sex- and age-matched controls. Standard lung function testing was performed (Vmax encore VE22d; Cardinal Health, Bilthoven, the Netherlands) after bronchodilation with $400 \mu \mathrm{g}$ salbutamol, according to standardised procedures [23]. Multiple breath washout (MBW) tests were performed in triplicate, with equipment and analysis methods for functional residual capacity (FRC) and LCI described in detail in a recent normative study; predicted values for LCI were also computed from the reference equation (applicable to both sexes) from that study [24]. Washout curvilinearity was determined in a similar way to that previously obtained in a recent physiological study [22]. Here, curvilinearity was computed as unity minus the ratio of two regression slopes obtained from the semilog plot of mean expired nitrogen concentration versus lung turnover (TO) down to the level where mean expired concentration reached 1/40th of the pre-test nitrogen concentration (and where LCI was determined). The regression slope in the denominator of the ratio of regression slopes is that determined in the range $\mathrm{TO}=0$ and $\mathrm{TO}=\mathrm{LCI} / 2$ and in the numerator that between $\mathrm{TO}=\mathrm{LCI} / 2$ and $\mathrm{TO}=\mathrm{LCI}$; the value for curvilinearity is zero in the case of a linear semilog plot and increases with 
curvilinearity up to 1 (corresponding to a hypothetical semilog plot with constant concentration beyond $\mathrm{TO}=\mathrm{LCI} / 2)$.

\section{Model simulations}

For comparison to experimental values, curvilinearity was also computed from a predicted washout curve based on the simplest possible conceptual model of ventilation heterogeneity: two lung compartments and no dead space. As has been shown by us and others [21,22], curvilinearity can help to identify which effect (dead space or specific ventilation difference) drives an increased LCI. While increased specific ventilation heterogeneity as well as increased dead space are expected to induce a slower decay of the washout nitrogen concentration curve and thus lead to LCI increases, the curvilinearity of the nitrogen washout curve is expected to be hardly affected by dead space $[21,22]$. Indeed, nitrogen washout curvilinearity arises when any two lung units with a different specific ventilation lead to a combined nitrogen washout curve that is the ventilation-weighted sum of two exponentials with a different washout rate. In summary, LCI is expected to increase with increased dead space and increased specific ventilation heterogeneity, whereas curvilinearity is expected to increase only with increased specific ventilation heterogeneity [22].

Washout concentration curves were considered for two plausible scenarios of ventilation heterogeneity. In the first scenario, a fixed part of the lung becomes increasingly less compliant: a fixed part of the lung, either $20 \%$ (scenario $1 \mathrm{a}$ ) or $50 \%$ (scenario $1 \mathrm{~b}$ ), gets a decreasing portion (100-10\%) of the nominal inspired volume that it would get in the case of normal expansion (where the nominal inspired volume received by each part of the lung is in proportion to its resting volume). In the second scenario, an increasingly greater part of the lung becomes less compliant: an increasing part of the lung (10-50\%) gets only a given percentage $(20 \%)$ of what it would get in the case of normal expansion.

The simple equations used for obtaining the washout concentrations, as well as the various cases of volume partitioning considered to cover the above conditions, are detailed in the online supplementary material. For one intermediate case of ventilation heterogeneity, similar simulations were carried out by considering either three or ten, instead of just two, compartments (see online supplementary material).

\section{Statistical analysis}

Unpaired t-tests, Mann-Whitney U-tests and Spearman rank correlations were performed (MedCalc; MedCalc Software bvba, Ostend, Belgium); statistical significance was accepted at a p-value $<0.05$.

\section{Results}

Table 1 summarises all relevant characteristics and lung function parameters under study in the CF patients and their matched controls. The CF patients were slightly shorter $(\mathrm{p}=0.03)$ and lighter $(\mathrm{p}=0.003)$ than the age- and sex-matched controls (by unpaired t-test). Of the lung function parameters, only the transfer coefficient of the lung for carbon monoxide and total lung capacity were not different from controls $(\mathrm{p}>0.1$ using the unpaired t-test). Figure 1 shows a significant association between FEV1 \% pred and LCI \% pred $(\mathrm{R}=-0.76 ; \mathrm{p}<0.001$ using the Spearman rank correction).

Figure 2 shows a typical washout curve obtained in a young male CF patient and his matched control. While the number of lung turnovers needed to reach the $1 / 40$ th threshold (LCI) was clearly increased in the CF patient, another striking feature of the washout curve is its curvilinearity (quantified here by curvilinearity). Figure 3 shows that the CF group as a whole had a significantly greater average LCI and curvilinearity value (table 1$)$, and that both were associated $(\mathrm{R}=0.67 ; \mathrm{p}<0.001$ using the Spearman rank correlation). In particular, the two out of 25 CF patients with normal LCI had normal curvilinearity values (fig. 3). The dashed line in figure $3 \mathrm{c}$ also indicates the predicted relationship between LCI and curvilinearity in the absence of any dead space; the actual curvilinearity and LCI numbers on which this prediction curve is based are given in table 2 (specific information for obtaining the prediction curve is given in the online supplementary material). In figure $3 c$, a fixed dead space would necessarily increase simulated LCI for a given curvilinearity [22], even in the control subjects. However, the magnitude of the dead space effect (including instrumental, anatomical and physiological dead space) is not simulated here and may not be constant in the entire curvilinearity range.

Figure 4 shows predicted washout concentration curves for $1 \mathrm{~L}$ tidal breathing with an FRC of $3 \mathrm{~L}$ and no dead space, in the case of two scenarios: a fixed part of the lungs becoming increasingly less compliant (scenariola, b), or, an increasingly greater part of the lung is less compliant (scenario 2). Figure 4 shows that scenarios 1 and 2 both generate increases in the intercept of the washout curve with a given threshold, potentially increasing LCI values. Figure 4 also shows a deviation from a straight line in these semilog washout plots, even when only $20 \%$ of the lungs is ventilated differently from the remainder of the lungs). However, in the latter case (fig. 4a), curvilinearity only really appears at very low concentrations: even when 
TABLE 1 Anthropometric, clinical and lung function data in cystic fibrosis (CF) and control groups

CF group $\# \quad$ Control group $\# \quad$ p-value

Male/female

Age years

Height $\mathrm{cm}$

Weight $\mathbf{k g}$

BMI $\mathrm{kg} \cdot \mathrm{m}^{-2}$

Clinical parameters

Pancreatic insufficiency

CFRD

Pseudomonas aeruginosa

F508del

Bronchiectasis

\section{Lung function}

FEV1 \% pred

FVC \% pred

FEV1/FVC \%

FEF25-75\% \% pred

DLCO \% pred

Kco \% pred

RV/TLC \%

TLC \% pred

Multiple breath washout

VT $\mathrm{mL}$

FRC $\mathrm{mL}$

VT/FRC

$\mathrm{LCl}$

LCl \% pred

Curvilinearity

\section{4/11}

28 (24-29)

$170 \pm 8$

$60 \pm 9$

$20.9 \pm 1.7$

24

8

24

7

24

$65 \pm 20$
$87 \pm 15$
$69(55-72)$
$23(12-27)$
$69 \pm 18$
$106 \pm 16$
$37 \pm 13$
$98 \pm 12$

$1125 \pm 65$

$2715 \pm 760$

$0.45 \pm 0.14$

$10.3 \pm 2.3$

$173 \pm 36$

$0.57 \pm 0.13$

\section{4/11}

27 (24-30)

$175 \pm 10$

$70 \pm 11$

$22.7 \pm 3.0$

0.03

0.003

0.012

Data are presented as $n$, mean \pm SD or median $(95 \% \mathrm{CI})$, unless otherwise stated. BMI: body mass index; CFRD: cystic fibrosis-related diabetes; FEV1: forced expiratory volume in $1 \mathrm{~s} ; \%$ pred: \% predicted; FVC: forced vital capacity; FEF25-75\%: forced expiratory flow at 25-75\% of FVC; DLCO: diffusing capacity of the lung for carbon monoxide; KCO: transfer coefficient of the lung for carbon monoxide; RV: residual volume; TLC: total lung capacity; VT: tidal volume; FRC: functional residual capacity; LCl: lung clearance index. ${ }^{\#}$ : for parameters failing normality test, data are presented as median $(95 \% \mathrm{Cl})$ and the Mann-Whitney U-test was used instead of the unpaired t-test to test for differences.

FIGURE 1 Scatterplots of lung clearance index (LCI) versus forced expiratory volume in $1 \mathrm{~s}$ (FEV1) for cystic fibrosis (CF) patients and matched controls. Spearman rank correlation for the $\mathrm{CF}$ group $\mathrm{R}=-0.76$ $(\mathrm{p}<0.001)$.

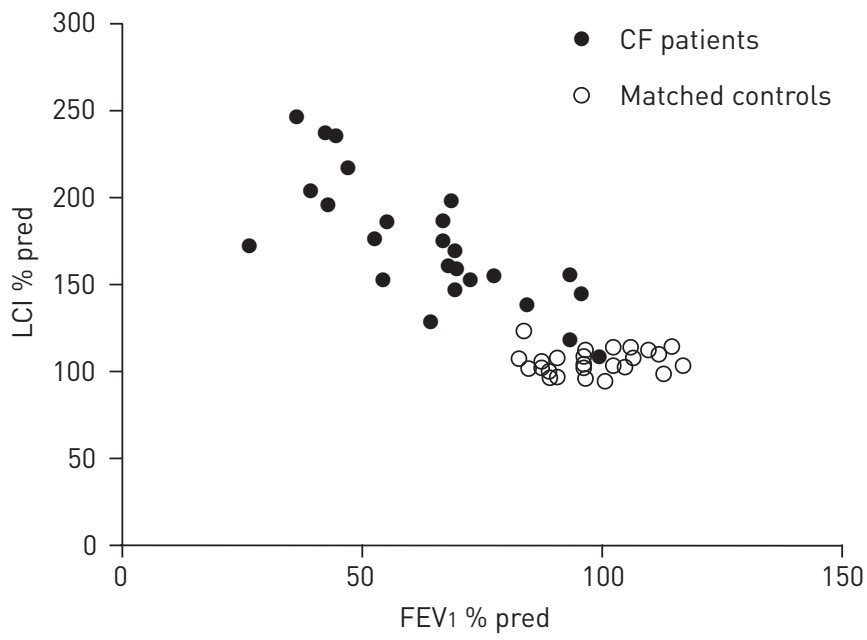




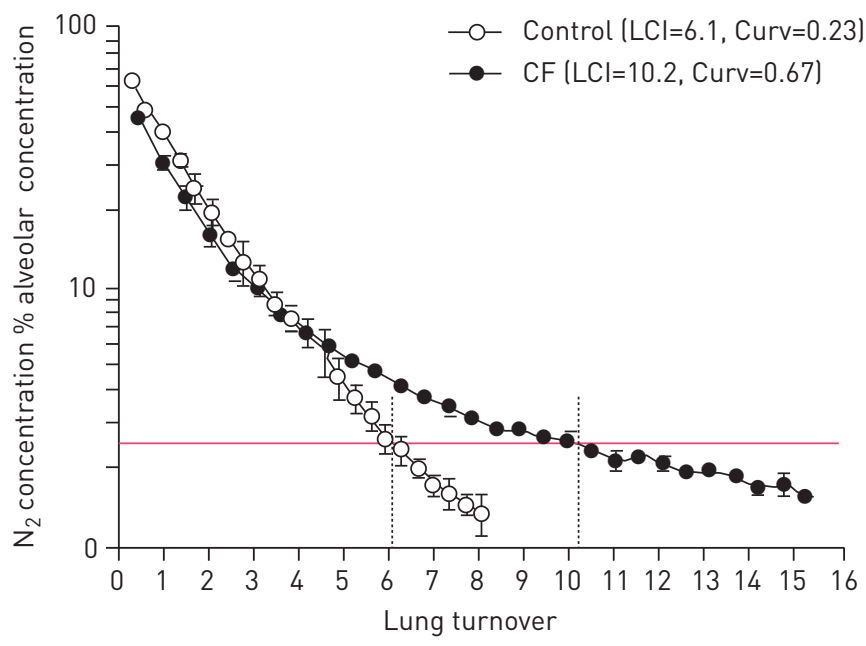

FIGURE 2 Typical mean expired nitrogen concentration curve, normalised to pretest nitrogen concentration and in semilog plot, for a 19-year-old male cystic fibrosis (CF) patient (forced expiratory volume in 1s (FEV1) 53\% predicted) and for the matched control subject (FEV1 83\% pred). Dotted lines indicate the lung clearance index (LCI) values (6.1 and 10.2); corresponding curvilinearity (Curv) values derived from these curves are 0.23 (control) and $0.67(\mathrm{CF})$.

$20 \%$ of the lung volume gets only $10 \%$ of the $20 \%$ inspired volume it should be getting (i.e. receiving only $2 \%$ of the inspired volume; largest solid circles), curvilinearity only really kicks in below the 1/40 level, and the curvilinearity value determined down to $1 / 40$ amounts to only 0.24 in this case of a relatively small, but severely underventilated, portion of the lung (table 2).

In contrast to LCI, curvilinearity values in table 2 corresponding to the washout curves in figure 4 can be directly compared with experimental values because dead space has little impact on curvilinearity. Besides the two CF patients with normal LCI and curvilinearity, all curvilinearity values in the CF group exceeded 0.40 . Table 2 shows that such a degree of curvilinearity can only be achieved if either at least half of the lungs gets only $40 \%$ of half the inspired volume it should be getting (scenario $1 \mathrm{~b}$ ) or if $30 \%$ of the lungs gets only $20 \%$ of the $30 \%$ inspired volume it should be getting (scenario 2 ). The maximum curvilinearity of $\sim 0.70$ observed in our CF patient group typically corresponds to at least half of the lungs getting only $20 \%$ of half the inspired volume it should be getting (based on scenario 1 b or 2 in table 2).

We briefly examined the effect of considering three or ten compartments instead of two on simulated washout curves and derived parameters LCI and curvilinearity. The results are found in online supplementary figure S3 and can be summarised as follows. First, the three-compartment simulation showed that the underventilated unit should represent a sizeable portion of the lungs for it to have an impact on the LCI. Secondly, for a given distribution of ventilation over a number of compartments (for instance, 10), it is possible to reduce it to an equivalent two-compartment model generating similar curvilinearity and LCI by considering, in one compartment, the average ventilation of the best ventilated units and, in the other compartment, the balance ventilation. Thirdly, the actual distribution of where the
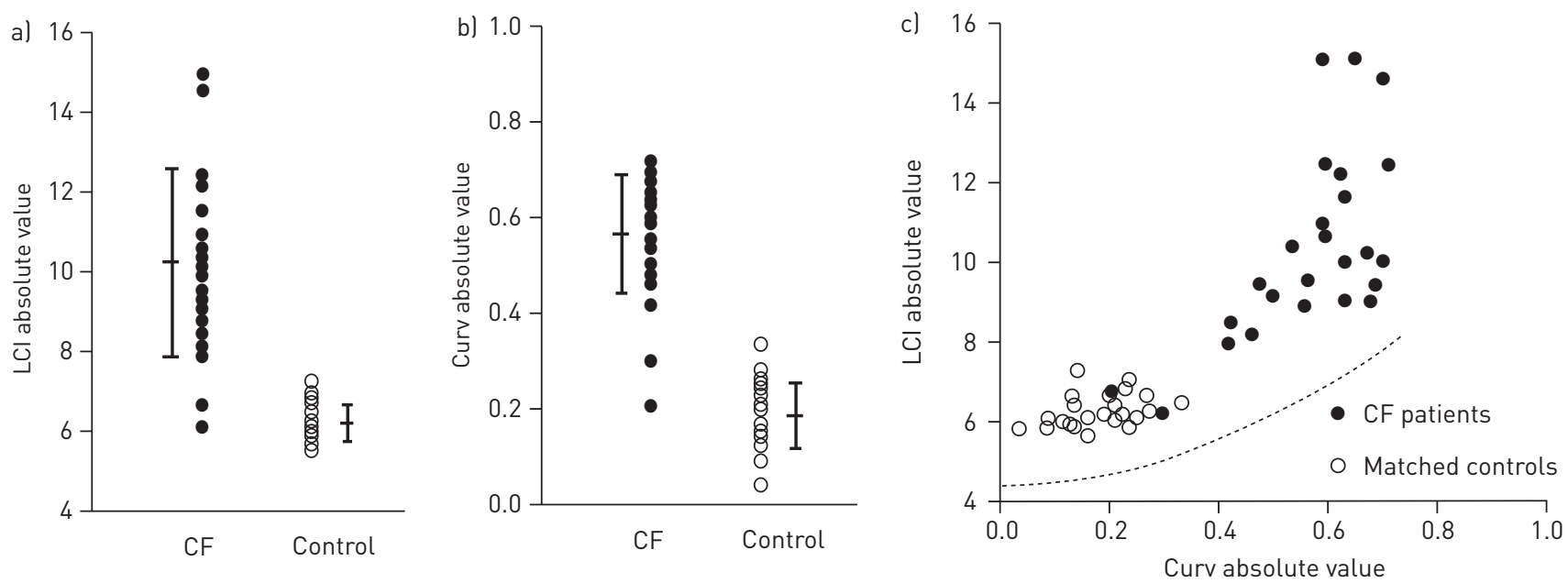

FIGURE 3 Absolute values for a) lung clearance index (LCI) and b) curvilinearity (Curv) in cystic fibrosis (CF) patients and matched controls. Data are presented as mean \pm SD. c) Scatterplot of LCI versus Curv in CF patients and matched controls, and the predicted relationship in the absence of any dead space (dashed line). 


\begin{tabular}{|c|c|c|c|c|}
\hline Cases & & Curv & $\mathrm{LCl}$ & $\mathrm{FRC} \mathrm{mL}$ \\
\hline \multicolumn{5}{|c|}{ Scenario $1 a^{\#}$} \\
\hline 1 & Homogeneous (100\%) & 0.00 & 4.3 & 3000 \\
\hline 2 & $50 \%$ & 0.12 & 4.5 & 2966 \\
\hline 3 & $40 \%$ & 0.17 & 4.7 & 2940 \\
\hline 4 & $30 \%$ & 0.23 & 4.8 & 2891 \\
\hline 5 & $20 \%$ & 0.28 & 5.0 & 2805 \\
\hline 6 & $10 \%$ & 0.24 & 4.9 & 2645 \\
\hline \multicolumn{5}{|c|}{ Scenario $1 b^{\top}$} \\
\hline 7 & Homogeneous (100\%) & 0.00 & 4.3 & 3000 \\
\hline 8 & $50 \%$ & 0.32 & 5.2 & 2931 \\
\hline 9 & $40 \%$ & 0.44 & 5.8 & 2891 \\
\hline 10 & $30 \%$ & 0.57 & 6.7 & 2824 \\
\hline 11 & $20 \%$ & 0.70 & 8.0 & 2693 \\
\hline 12 & $10 \%$ & 0.85 & 9.1 & 2304 \\
\hline \multicolumn{5}{|c|}{ Scenario $2^{+}$} \\
\hline 13 & Homogeneous $(0 \%)$ & 0.00 & 4.3 & 3000 \\
\hline 14 & $10 \%$ & 0.12 & 4.6 & 2898 \\
\hline 15 & $20 \%$ & 0.28 & 5.0 & 2805 \\
\hline 16 & $30 \%$ & 0.49 & 5.7 & 2731 \\
\hline 17 & $40 \%$ & 0.65 & 6.8 & 2692 \\
\hline 18 & $50 \%$ & 0.70 & 8.0 & 2693 \\
\hline
\end{tabular}

Note that cases (5) and (15), and cases (11) and (18) are identical. Curv, LCl, and FRC were determined considering the portion of the washout curve down to the threshold of $2.5 \%$ of the initial alveolar concentration. ${ }^{\#}$ : $20 \%$ of the lung volume gets variable inspired volume $(10-50 \% ; 100 \%) ;{ }^{\top}: 50 \%$ of the lung volume gets variable inspired volume $(10-50 \% ; 100 \%) ;{ }^{+}$: a variable part of lung volume $(0 \% ; 10-50 \%)$ gets $20 \%$ of nominal inspired volume.

best and least ventilated units are located is immaterial to curvilinearity or LCI. Finally, we made some additional simulations with the two-compartment model to verify the presence of the local maxima in curvilinearity and LCI values when one unit gets severely underventilated (obtained for case 5 in scenario 1a) (table 2). In fact, this was found to be consistent with the fact that further decrease of ventilation of the least ventilated compartment would lead to the limit case of a homogeneously ventilated lung. Details on the above simulations can be found in online supplementary figure S2.
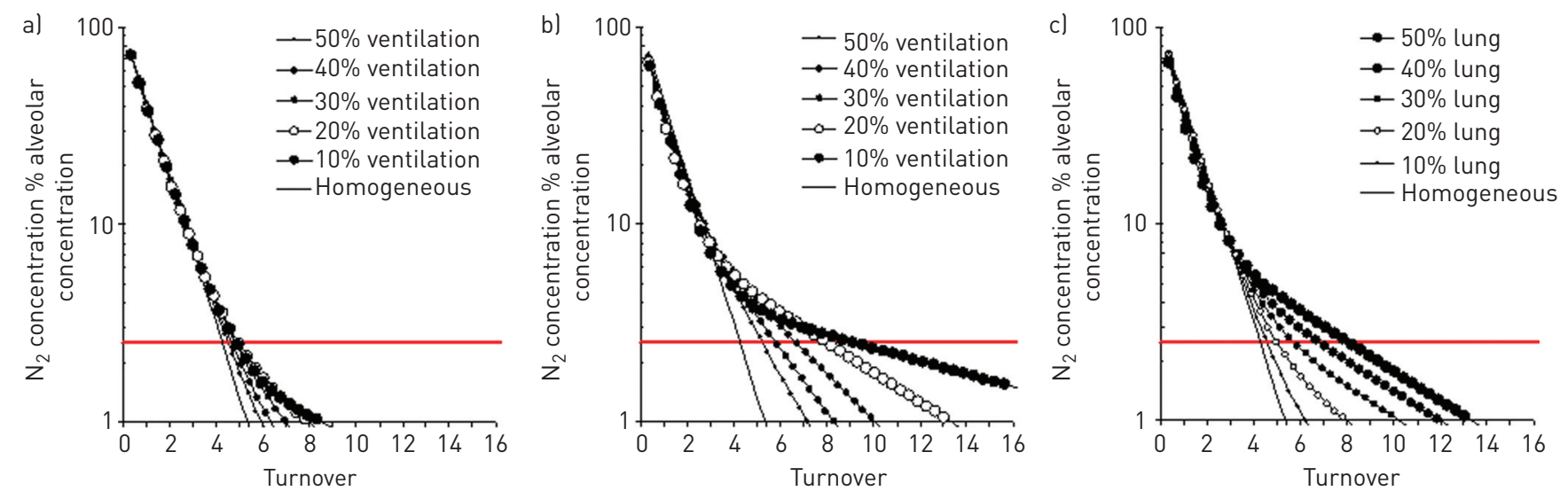

FIGURE 4 Predicted washout concentration curves for a and b) scenario 1 and c) scenario 2 with corresponding functional residual capacity (FRC), lung clearance index (LCI) and curvilinearity values in table 2. A fixed part a) $20 \%$ and b) $50 \%$ of the lung gets a decreasing portion (100-10\%) of what it would get in the case of normal expansion. c) An increasing part of the lung (10-50\%) gets only a given percentage (20\%) of what it would get in the case of normal expansion. Note that the open circles in a) and c) represent the same curve, resulting from $20 \%$ of the lung receiving $20 \%$ of the inspired volume it would get in case of normal expansion. 


\section{Discussion}

Beside the potential of LCI to reflect ventilation heterogeneities beyond the resolution of standard lung function and imaging techniques, a major determinant of the LCI abnormality in adult CF lung disease is shown here to be operational between relatively large lung units that are supplied by convective flow. This confirms early data by BROWN et al. [25] who used aerosol distribution as a surrogate marker for convective ventilation distribution in CF patients with a very similar FEV1 and age range. In our CF patients, LCI correlated with FEV1 (fig. 1), providing a first indication that the poorly ventilated units that delay the efficiency of gas mixing also contribute to a considerable amount of the expiratory flow limitation. To explain the different information content of FEV1 and LCI despite some degree of correlation, AURORA et al. [2] pointed out that parallel heterogeneities of specific ventilation preferentially lead to LCI increases. While this is the case for both LCI and curvilinearity, the former is also increased by an increase in dead space, which explains the consistently greater experimental LCI than that predicted by convection-dependent ventilation heterogeneities reflected in curvilinearity (fig. 3c). As dead space includes the physiological dead space due to intra-acinar heterogeneities, it is almost impossible to link this portion of LCI to morphometry of most peripheral air spaces in a meaningful quantitative way in adult CF patients. In the high LCI range, there appear to be some patients (with an LCI >12) for whom a relatively greater portion of LCI cannot be explained by convection-dependent ventilation heterogeneities.

The curvilinearity of the washout concentration curves does offer a possibility to obtain a semi-quantitative handle on the magnitude of dysfunctional convection-dependent lung units. In adult CF, curvilinearity was much greater than that previously observed in normal subjects after bronchoprovocation or in adult asthma patients, where curvilinearity never exceeded 0.40 [22]. Apart from two CF patients with normal LCI and curvilinearity values, experimental curvilinearity values ranged from 0.40 to 0.70 , which corresponds to $30 \%$ to $50 \%$, respectively, of total lung volume that is likely to be ventilated to a lesser extent than the remainder of the lungs (table 2). It is important to realise that the conceptual model on which this estimation is based, does not make any assumptions about the proximity of the poorly ventilated units. The 10-compartment model explicitly shows this by means of two equivalent distributions of poorly and well ventilated units (online supplementary figure S3b and c) and, in the two-compartment model, the poorly ventilated units are jointly represented by the least ventilated of the two compartments. Hence, it is possible that the poorly ventilated units are distributed all over the lung, as one could imagine in a case where such lung units correspond to lung volumes subtending from bronchiectatic airways scattered across the lungs. Whatever the exact origin of the poor ventilation to certain portions of the lung, our curvilinearity data suggest that, cumulatively, these lung units can make up $>30 \%$ of lung volume in adult CF patients. We also illustrated that extending the conceptual model to more than two compartments does not a priori provide additional information. An extension to more compartments can only be of incremental value when the various compartments can be attributed realistic volumes and ventilation rates (e.g. based on dynamic imaging modalities).

It is tempting to suggest that our MBW data are compatible with the very patchy ventilation images obtained in adult CF patients by hyperpolarised helium-3 magnetic resonance imaging ( $\left.{ }^{3} \mathrm{He}-\mathrm{MRI}\right)$ [26-29], where the extent of ventilation defects correlated with FEV1 [26-28]. When the extent of ventilation defects (i.e. underventilated lung zones with a signal intensity below the imaging threshold) was expressed as percentage lung volume, this averaged $26 \%$ in CF patients with FEV1 ranging 27-93\% pred [27] and 17$20 \%$ in CF patients with FEV1 ranging $50-96 \%$ pred [28]. If, in addition to unventilated lung, the hypo-, normal and hyperventilated lung volumes are also considered, only $\sim 30 \%$ of the lung was ventilated normally [29]. Unfortunately, access to such imaging modalities that can provide three-dimensional ventilation maps is limited. CT is more readily available and, in one imaging study [27], in CF patients' scores from MRI and HRCT were shown to correlate, with bronchiectasis as the only independent predictor of ${ }^{3} \mathrm{He}$-MRI. Hence, it can be expected that curvilinearity (and thus LCI) would correlate with the degree of bronchiectasis as a surrogate marker for the underventilated lung volumes they subtend, bearing in mind that central bronchiectatic airways subtend a larger volume, potentially accounting for relatively more of the observed curvilinearity (or LCI). Standardised image analysis did lead to correlations between LCI and CT scores of bronchiectasis $[15,16]$. In one study, LCI and CT scores correlated despite being collected at different time-points within a 6-month time frame [16], which suggests that that LCI at least partly reflects a fixed or slowly evolving, and possibly irreversible, component of structural alteration in these patients.

Given the extent of ventilation heterogeneity observed here, and the large cumulative lung volume involved, it is unlikely that any short-term intervention exists to restore either curvilinearity or LCI to normal. This possibly explains why the gain in LCI following various short-term interventions has been limited [6-10]; however, this should not be a reason to discard the use of LCI in adult CF patients altogether. It would be interesting to observe longitudinally the washout curves leading up to such a degree of ventilation heterogeneity, according to the scenarios displayed across figure 4, as part of the lung becomes increasingly 
affected (fig. $4 \mathrm{a}$ and $\mathrm{b}$ ) or as the affected portion of the lungs increases in size (fig. 4c), or a combination of both. Of the many MBW indices [21], LCI has been advocated as the preferred outcome measure, citing a comprehensive link to curves such as those in figure 4 and its sensitivity in early CF [30]. Certainly, curvilinearity also stands the test of being comprehensive, with curvilinearity $=0$ and 1 representing a similar ventilation of all lung units and the presence of an infinitesimally slowly emptying lung unit, respectively. In advanced CF at least, curvilinearity is shown to reveal quantitative information about largescale ventilation heterogeneities that would not otherwise be gained from LCI alone.

Simultaneous inspection of LCI and curvilinearity could also indicate whether or not any interventional or longitudinal changes can be attributed preferentially to small airways dysfunction within convectiondependent lung units. In the former case, the curvilinearity would remain essentially unaffected and one could then speculate that an increased LCI mainly reflects intra-acinar ventilation heterogeneities. However, when decreased specific ventilation develops in certain portions of the lung, both LCI and curvilinearity will increase. When the dysfunctional portion of the lung is $\leqslant 20 \%$, curvilinearity will only appear at very low concentrations $(<1 / 40$ th initial concentration), where experimental accuracy becomes a limiting factor (fig. 4a). Importantly, if curvilinearity and LCI are to represent the various aspects of ventilation heterogeneities happening at the same time, one must consider LCI at one threshold level (e.g. 1/40) and curvilinearity down to that same threshold. In this way, both parameters represent all ventilation heterogeneities participating in the gas mixing processes occurring down to the $1 / 40$ threshold level, as was performed here. Finally, the use of a reference point, such as the 1/40 threshold for FRC estimation, is also important in the interpretation of paradoxical LCI increases following treatment [8]. It has been suggested that treatment can lead to newly recruited portions of the lung participating in the gas mixing process, potentially rendering it more heterogeneous, and thus paradoxically increasing LCI. If such argumentation is to be made in a semiquantitative way, the FRC estimate determined at a fixed threshold will play a direct role (estimating the amount of newly recruited lung volume), as well as an indirect one (via its effect on LCI computation).

\section{Limitations of the study}

The present study of ventilation heterogeneities in CF has two main limitations. First, there is no actual simulation of the dead space contribution to LCI. For instance, it is possible that in the curvilinearity range, around and above 0.6 in figure $3 c$, data-points corresponding to LCI $>12$ are due to an increased contribution from the intra-acinar dead space with respect to its contribution in the curvilinearity range $<0.6$. However, in the absence of any morphometrical information on the type and extent of airway changes at the level of the acinar lung zone, it is impossible to produce a reasonable simulation of this contribution. The dotted line merely provides an indication of how much of the LCI can be accounted for by convection-dependent ventilation heterogeneities. Secondly, we do not have imaging data in the same patients in search of a direct link between the convection-dependent (i.e. relatively large-scale) ventilation heterogeneities derived from the MBW test. While the best direct comparison to be made is with ventilation imaging (MRI), the underlying structural changes potentially responsible for the ventilation defects could provide a structure-function link (HRCT). The success of relating MBW indices to either imaging modality also implies advanced image-processing tools that actually segment and quantify the volumes corresponding to underventilated lung zones (MRI) or estimate the lung volumes supplied by the affected airways (HRCT).

In conclusion, if the MBW test is to be reduced to a few parameters to facilitate a posteriori quantitative analyses, the parameter of choice, in addition to LCI, is the washout curvilinearity. The latter is dominated by differences in specific ventilation between lung units, and poorly affected by dead spaces generated by gas mixing inefficiency within these lung units or by series dead space. While dead spaces other than instrumental dead space are difficult to quantify in order to obtain a valid LCI, washout curvilinearity can be more readily estimated by simple computations with zero dead space. These show that in the majority of our adult CF patients, at least $30 \%$ of the lungs must be ventilated to a different extent than the remainder of the lungs.

\section{References}

1 Gustafsson PM, Aurora P, Lindblad A. Evaluation of ventilation maldistribution as an early indicator of lung disease in children with cystic fibrosis. Eur Respir J 2003; 22: 972-979.

2 Aurora P, Gustafsson P, Bush A, et al. Multiple breath inert gas washout as a measure of ventilation distribution in children with cystic fibrosis. Thorax 2004; 59: 1068-1073.

Horsley A. Lung clearance index in the assessment of airways disease. Respir Med 2009; 103: 793-799.

4 Aurora P, Stanojevic S, Wade A, et al. Lung clearance index at 4 years predicts subsequent lung function in children with cystic fibrosis. Am J Respir Crit Care Med 2011; 183: 752-758.

5 Kraemer R, Blum A, Schibler A, et al. Ventilation inhomogeneities in relation to standard lung function in patients with cystic fibrosis. Am J Respir Crit Care Med 2005; 171: 371-378. 

2007; 42: 168-176.

7 Fuchs SI, Toussaint S, Edlhaimb B, et al. Short-term effect of physiotherapy on variability of the lung clearance index in children with cystic fibrosis. Pediatr Pulmonol 2010; 45: 301-306.

8 Robinson PD, Cooper P, Van Asperen P, et al. Using index of ventilation to assess response to treatment for acute pulmonary exacerbation in children with cystic fibrosis. Pediatr Pulmonol 2009; 44: 733-742.

9 Amin R, Subbarao P, Lou W, et al. The effect of dornase alfa on ventilation inhomogeneity in patients with cystic fibrosis. Eur Respir J 2011; 37: 806-812.

10 Amin R, Subbarao P, Jabar A, et al. Hypertonic saline improves the LCI in paediatric patients with CF with normal lung function. Thorax 2010; 65: 379-383.

11 Horsley AR, Gustafsson PM, Macleod KA, et al. Lung clearance index is a sensitive, repeatable and practical measure of airways disease in adults with cystic fibrosis. Thorax 2008; 63: 135-140.

12 Konstan MW, Wagener JS, Vandevanter DR, et al. Risk factors for rate of decline in FEV1 in adults with cystic fibrosis. J Cyst Fibros 2012; 11: 405-411.

13 Robinson PD, Goldman MD, Gustafsson PM. Inert gas washout: theoretical background and clinical utility in respiratory disease. Respiration 2009; 78: 339-355.

14 Gustafsson PM, De Jong PA, Tiddens HA, et al. Multiple-breath inert gas washout and spirometry versus structural lung disease in cystic fibrosis. Thorax 2008; 63: 129-134.

15 Owens CM, Aurora P, Stanojevic S, et al. Lung clearance index and HRCT are complementary markers of lung abnormalities in young children with CF. Thorax 2011; 66: 481-488.

16 Ellemunter H, Fuchs SI, Unsinn KM, et al. Sensitivity of lung clearance index and chest computed tomography in early CF lung disease. Respir Med 2010; 104: 1834-1842.

17 de Jong PA, Lindblad A, Rubin L, et al. Progression of lung disease on computed tomography and pulmonary function tests in children and adults with cystic fibrosis. Thorax 2006; 61: 80-85.

18 Singer F, Stern G, Thamrin C, et al. A new double-tracer gas single-breath washout to assess early cystic fibrosis lung disease. Eur Respir J 2013; 41: 339-345.

19 Van Muylem A, Baran D. Overall and peripheral inhomogeneity of ventilation in patients with stable cystic fibrosis. Pediatr Pulmonol 2000; 30: 3-9.

20 Horsley AR, Macleod KA, Robson AG, et al. Effects of cystic fibrosis lung disease on gas mixing indices derived from alveolar slope analysis. Respir Physiol Neurobiol 2008; 162: 197-203.

21 Engel LA. Intraregional gas mixing and distribution. In: Engel LA, Paiva M, eds. Gas Mixing and Distribution in the Lung. New York, Marcel Dekker, 1985; pp. 287-358.

22 Verbanck S, Paiva M, Schuermans D, et al. Relationships between the lung clearance index and conductive and acinar ventilation heterogeneity. J Appl Physiol 2012; 112: 782-790.

23 Pellegrino R, Viegi G, Brusasco V, et al. Interpretative strategies for lung function tests. Eur Respir J 2005; 26: 948-968.

24 Verbanck S, Thompson BR, Schuermans D, et al. Ventilation heterogeneity in the acinar and conductive zones of the normal ageing lung. Thorax 2012; 67: 789-795.

25 Brown JS, Gerrity TR, Bennett WD. Effect of ventilation distribution on aerosol bolus dispersion and recovery. J Appl Physiol 1998; 85: 2112-2117.

26 Mentore K, Froh DK, de Lange EE, et al. Hyperpolarized HHe 3 MRI of the lung in cystic fibrosis: assessment at baseline and after bronchodilator and airway clearance treatment. Acad Radiol 2005; 12: 1423-1429.

27 McMahon CJ, Dodd JD, Hill C, et al. Hyperpolarized ${ }^{3}$ helium magnetic resonance ventilation imaging of the lung in cystic fibrosis: comparison with high resolution CT and spirometry. Eur Radiol 2006; 16: $2483-2490$.

28 Kirby M, Svenningsen S, Ahmed H, et al. Quantitative evaluation of hyperpolarized helium-3 magnetic resonance imaging of lung function variability in cystic fibrosis. Acad Radiol 2011; 18: 1006-1013.

29 Kirby M, Heydarian M, Svenningsen S, et al. Hyperpolarized ${ }^{3} \mathrm{He}$ magnetic resonance functional imaging semiautomated segmentation. Acad Radiol 2012; 19: 141-152.

30 Robinson PD, Lindblad A, Gustafsson PM. Comparison of the utility of multiple breath inert gas washout parameters in cystic fibrosis. Thorax 2010; 65: 659. 